\title{
A Novel Electrochemical Sensor for Probing Doxepin Created on a Glassy Carbon Electrode Modified with Poly(4-Amino- benzoic Acid)/Multi-Walled Carbon Nanotubes Composite Film
}

\author{
Xiao-Li Xu, Fei Huang, Guo-Liang Zhou, Song Zhang * and Ji-Lie Kong * \\ Department of Chemistry \& Center of Analysis and Measurement, Fudan University, \\ Handan Road 220\#, Shanghai 200433, China; E-Mails: 071022016@ fudan.edu.cn (X.-L.X.); \\ 041022013@fudan.edu.cn (F.H.); 072022057@fudan.edu.cn (G.-L.Z.) \\ * Author to whom correspondence should be addressed; E-Mails: songzhang@fudan.edu.cn (S.Z.); \\ jlkong@fudan.edu.cn (J.-L.K.); Tel.: +86-21-6564-2405; Fax: +86-21-6564-1740.
}

Received: 7July 2010; in revised form: 31 July 2010 / Accepted: 7 September 2010 /

Published: 8 September 2010

\begin{abstract}
A novel electrochemical sensor for sensitive detection of doxepin was prepared, which was based on a glassy carbon electrode modified with poly(4-aminobenzoic acid)/multi-walled carbon nanotubes composite film [poly(4-ABA)/MWNTs/GCE]. The sensor was characterized by scanning electron microscopy and electrochemical methods. It was observed that poly(4-ABA)/MWNTs/GCE showed excellent preconcentration function and electrocatalytic activities towards doxepin. Under the selected conditions, the anodic peak current was linear to the logarithm of doxepin concentration in the range from $1.0 \times 10^{-9}$ to $1.0 \times 10^{-6} \mathrm{M}$, and the detection limit obtained was $1.0 \times 10^{-10} \mathrm{M}$. The poly(4-ABA)/MWNTs/GCE was successfully applied in the measurement of doxepin in commercial pharmaceutical formulations, and the analytical accuracy was confirmed by comparison with a conventional ultraviolet spectrophotometry assay.
\end{abstract}

Keywords: poly(4-aminobenzoic acid); multi-walled carbon nanotubes; nanocomposite; doxepin; detection 


\section{Introduction}

Doxepin (Figure 1) has been widely used as an effective tricyclic antidepressant in the treatment of psychiatric disorders over the past decades [1,2]. Due to its importance, many analytical methods have been developed for its determination. Reported literature methods mainly focus on chromatographic techniques, such as reversed-phase liquid chromatography with ultraviolet detection [3], thin-layer chromatography-densitometry method [4], HPLC or LC-MS [5-8], and capillary zone electrophoresis [9], etc. Other methods are also available, including electrogenerated chemiluminescence [10], resonance light scattering [11], and the flow-injection techniques [12,13], etc. However, these methods are expensive, time-consuming and laborious.

Figure 1. Molecular structure of doxepin.

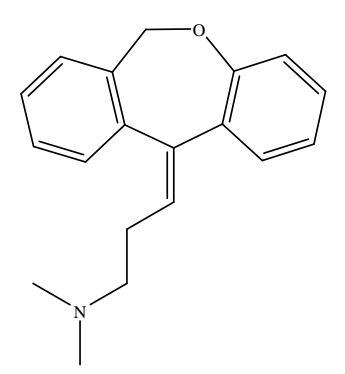

Electrochemical methods provide an attractive alternative way to determine doxepin due to their simplicity, sensitivity, speed and low costs. There are only a few literature reports investigating the electrochemical properties of doxepin. Among the few examples, polarography [14], ion-selective membrane electrode [15] and boron-doped diamond electrode [16] were all used to study doxepin. Nevertheless, their applications in routine analysis of doxepin might be restricted due to toxicity of the dropping mercury electrode, sluggish response and unsatisfactory detection limit. Therefore, it is necessary to devise new determination procedures for doxepin.

Carbon nanotube (CNT)-modified electrodes have been successfully applied to study antidepressants [17], owing to the excellent conductivity and catalytic activity of CNTs. On the other hand, conductive polymers (CPs), with unique high stability and selectivity characteristics, good conductivity and reproducibility, more active sites and good homogeneity, have also been used to detect antidepressants [18-20]. These electrodes functionalized solely with CNTs or CPs presented excellent accumulation and electrocatalytic activities toward the targets.

Recently, electrodes functionalized with nanocomposite films of CNTs and CPs have been reported to determine the antidepressant trifluoperazine [21], for which a more sensitive signal response, faster response and lower detection limit could be obtained, compared with the CNT or CP film-modified electrodes. There is no report about the electrochemical determination of doxepin by CP/CNT composite film-modified electrodes.

In this paper, a new nanocomposite poly(4-ABA) and MWNT material was prepared by electrochemical polymerization. The poly(4-ABA)/MWNTs/GCE was characterized and applied to the detection of doxepin under optimum conditions. Analytical conditions, such as amount of MWNTs, electropolymerization conditions, $\mathrm{pH}$ and buffer solution, accumulation time and potential, were optimized. 


\section{Experimental Section}

\subsection{Reagents}

Doxepin hydrochloride $\left(\mathrm{C}_{19} \mathrm{H}_{21} \mathrm{NO} \cdot \mathrm{HCl}\right)$ and its drug tablets were kindly supplied by Sine JiuFu Pharmaceutical Company (Shanghai, China). Doxepin stock solution $(0.01 \mathrm{M})$ was prepared with double distilled water and stored at $+4{ }^{\circ} \mathrm{C}$ in the dark. Buffer solution was $0.10 \mathrm{M}$ phosphate buffer solution (PBS, pH 6.2). MWNTs (diameter 10-30 nm, length 0.5-40 $\mu \mathrm{m}$ ) were supplied by Shenzhen Nanotech Port Co. Ltd. (Shenzhen, China). Before use, the received MWNTs were refluxed in a mixture of concentrated $\mathrm{H}_{2} \mathrm{SO}_{4}$ and $\mathrm{HNO}_{3}$ for about $6 \mathrm{~h}$, then washed with double distilled water and dried under vacuum at room temperature. A suspension of MWNTs was prepared by dispersing MWNTs $(2 \mathrm{mg})$ in $\mathrm{N}, \mathrm{N}$-dimethylformamide $(2 \mathrm{~mL})$ with the aid of ultrasonic agitation. 4-Aminobenzoic acid (4- $\mathrm{NH}_{2}-\mathrm{C}_{6} \mathrm{H}_{4}-\mathrm{CO}_{2} \mathrm{H}$, 4-ABA) was purchased from Sinopharm Chemical Reagent Co., Ltd. $1.0 \times 10^{-4} \mathrm{M} 4-\mathrm{ABA}$ solution was prepared with $0.10 \mathrm{M}$ PBS ( $\mathrm{pH} 7.5$ ), and was stored at $+4{ }^{\circ} \mathrm{C}$ in the dark. Before measurement, doxepin tablets were ground into powder, dissolved in water, filtered and diluted to a fixed volume. All the other chemicals were of analytical grade and used as received. Double distilled water was used exclusively in the experiments.

\subsection{Apparatus}

Electrochemical measurements were carried out on a CHI1030 multichannel voltammetric analyzer (ChenHua Instrument Company, Shanghai, China). The conventional three-electrode system was employed, including a platinum wire as counter electrode, a saturated calomel electrode (SCE) as reference electrode, and a bare $\operatorname{GCE}(\Phi=2.5 \mathrm{~mm})$, poly $(4-\mathrm{ABA}) / \mathrm{GCE}$, MWNTs/GCE, and poly(4-ABA)/MWNTs/GCE as working electrode. Electrochemical impedance spectroscopy (EIS) measurements were performed on an EG\&G PAR Model 273 A bipotentiostat (Princeton, NJ, USA) in conjunction with a lock-in amplifier. A 5-mV amplitude sine wave was applied to the electrode under potentiostatic control and the frequency range was from $0.05 \mathrm{~Hz}$ to $10 \mathrm{KHz}$. Scanning electron microscopy was performed on a Philips XL30 Microscope (Japan). UV-vis absorption spectra were obtained on an HP8453 ultraviolet-visible spectrophotometer (Agilent, Palo Alto, CA, USA).

\subsection{Fabrication of the poly(4-ABA)/MWNTs/GCE}

The nanocomposite film modified electrode was fabricated as follows: firstly, the bare GCE was polished sequentially with 0.3 and $0.05 \mu \mathrm{m} \mathrm{Al}_{2} \mathrm{O}_{3}$ slurry to form a mirror-like surface, then rinsed and ultrasonicated for $10 \mathrm{~min}$ in water, acetone, 1:1 $\mathrm{HNO}_{3}$ and water, respectively, and dried in air. The MWNTs/GCE was fabricated by dropping MWNT suspension $(8 \mu \mathrm{L})$ on the cleaned GCE surface and evaporating the solvent in air. Secondly, the resulting MWNTs/GCE was immersed in 0.10 M PBS $(\mathrm{pH} 7.5)$ containing $1.0 \times 10^{-4} \mathrm{M} 4-\mathrm{ABA}$, and cyclic voltammetry was performed from -1.4 to $+2.4 \mathrm{~V}$ for 10 cycles at a scan rate of $100 \mathrm{mV} \mathrm{s}^{-1}$ according to the literature [22]. Thus, the poly(4-ABA)/MWNTs/GCE was prepared. Before use, the modified electrode was continuously cycled from +0.3 to $+1.2 \mathrm{~V}$ in a blank buffer solution of $0.10 \mathrm{M}$ PBS (pH 6.2) until a stable cyclic voltammogram was obtained. All experiments were performed at about $25{ }^{\circ} \mathrm{C}$. 


\section{Results and Discussion}

\subsection{Cyclic voltammograms of electropolymerization of 4-ABA and characterization of the modified GCE}

The electropolymerization of $1.0 \times 10^{-4} \mathrm{M} 4-\mathrm{ABA}$ in $0.10 \mathrm{M}$ PBS (pH 7.5) at MWNTs/GCE is given in the repetitive cyclic voltammograms of Figure 2. As seen, in the first scan, an anodic peak 1 at about $1.37 \mathrm{~V}$ and a cathodic peak 2 at around $-0.73 \mathrm{~V}$ appeared, respectively. From the second cycle on, another anodic peak 3 occurred at about $0.09 \mathrm{~V}$. As the scans continued, the current of each peak increased, suggesting a continuous growth of the poly(4-ABA) film. Meanwhile, the current grew quickly in the initial five cycles, and then increased slowly until 10 scans. After the 10 scans, a thin adherent brown film could be found on the electrode surface. The electrochemical behavior of 4-ABA at MWNTs/GCE might be as follows [22,23]: 4-ABA was oxidized to free radical (peak 1) at first; the free radicals combined together rapidly to hydrazobenzoic acid; then hydrazobenzoic acid was oxidized to azobenzoic acid (peak 3), and azobenzoic acid reduced to hydrazobenzoic acid (peak 2).

Figure 2. Repetitive cyclic voltammograms of $1.0 \times 10^{-4} \mathrm{M} 4-\mathrm{ABA}$ at MWNTs/GCE. The insert is the first cycle of the cyclic voltammogram. Scan rate: $100 \mathrm{mV} \mathrm{s}^{-1}$; supporting electrolyte: 0.10 M PBS (pH 7.5).

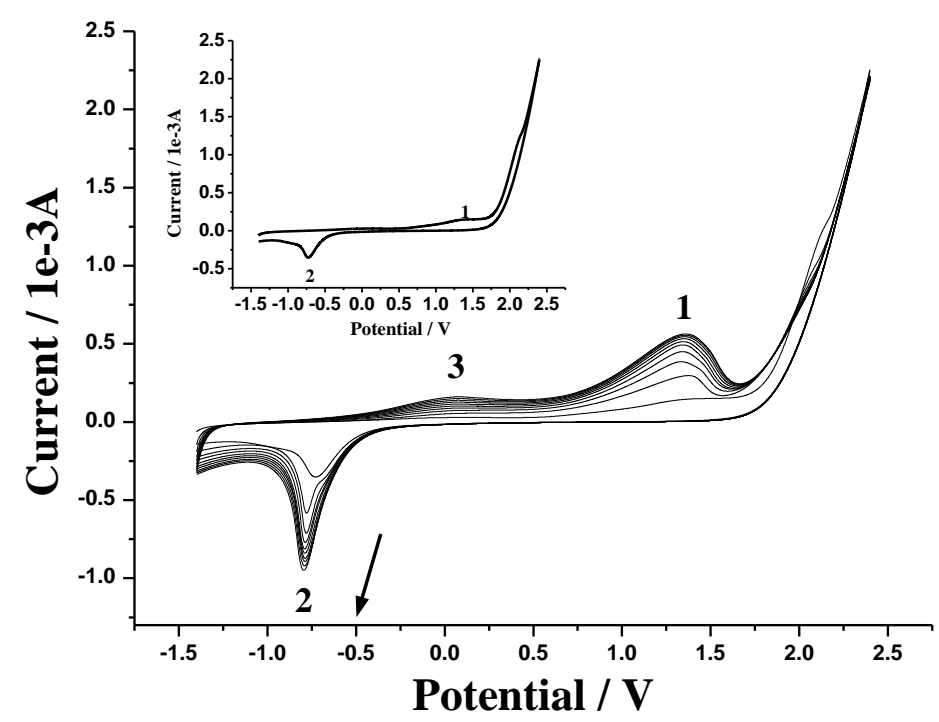

The surface morphology of MWNTs/GCE and poly(4-ABA)/MWNTs/GCE was investigated by scanning electron microscopy. As may be seen in Figure 3, MWNTs were well dispersed on the surface of the GCE (Figure 3A). After 4-ABA was polymerized in and on the MWNTs matrix, a stable, uniform, porous and three-dimensional film structure was formed (Figure 3B), which would presumably exhibit excellent interaction with doxepin molecules. It could be inferred that, the nanocomposite film might get more stable than the sole MWNTs film, as the thin layer of poly(4-ABA) could help fix the modified MWNTs and prevent them from leaving the electrode surface. 
Figure 3. The surface images of MWNTs/GCE (A) and poly(4-ABA)/MWNTs/GCE (B) produced by scanning electron microscopy. Scale bar: $1 \mu \mathrm{m}$.

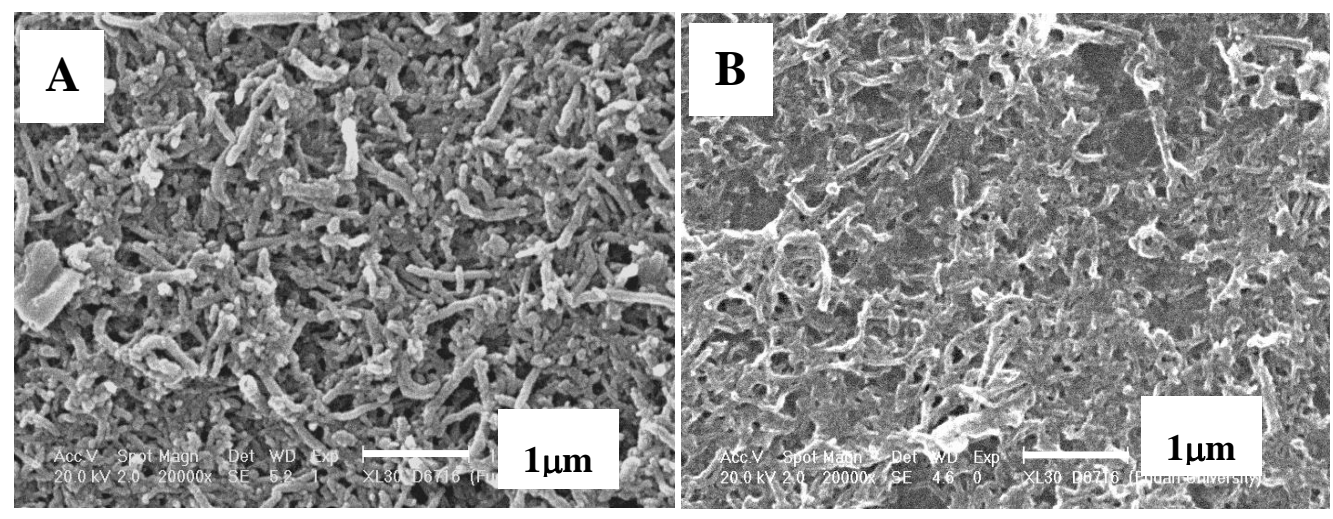

Electrochemical responses of $\mathrm{K}_{3} \mathrm{Fe}(\mathrm{CN})_{6}$ at the bare GCE and the modified electrodes are shown in Figure 4. The largest peak current and background current at the poly(4-ABA)/MWNTs/GCE (Figure 4D) were obtained when compared with those at the MWNTs/GCE, poly(4-ABA)/GCE, and bare GCE, which indicates the composite effect of MWNTs and poly(4-ABA) might benefit the electron transfer of the electrochemical probe, $\mathrm{Fe}(\mathrm{CN})_{6}{ }^{3-}$. Moreover, the clear increase of peak current at the MWNTs/GCE (Figure 4B) compared with that of the bare GCE (Figure 4A), also suggests the prominent conductivity of MWNTs.

Figure 4. Cyclic voltammograms of $5 \mathrm{mM} \mathrm{K}_{3} \mathrm{Fe}(\mathrm{CN})_{6}$ at bare $\mathrm{GCE}(\mathrm{A})$; MWNTs/GCE (B); poly(4-ABA)/GCE (C) and poly(4-ABA)/MWNTs/GCE (D). Scan rate: $100 \mathrm{mV} \mathrm{s}^{-1}$.

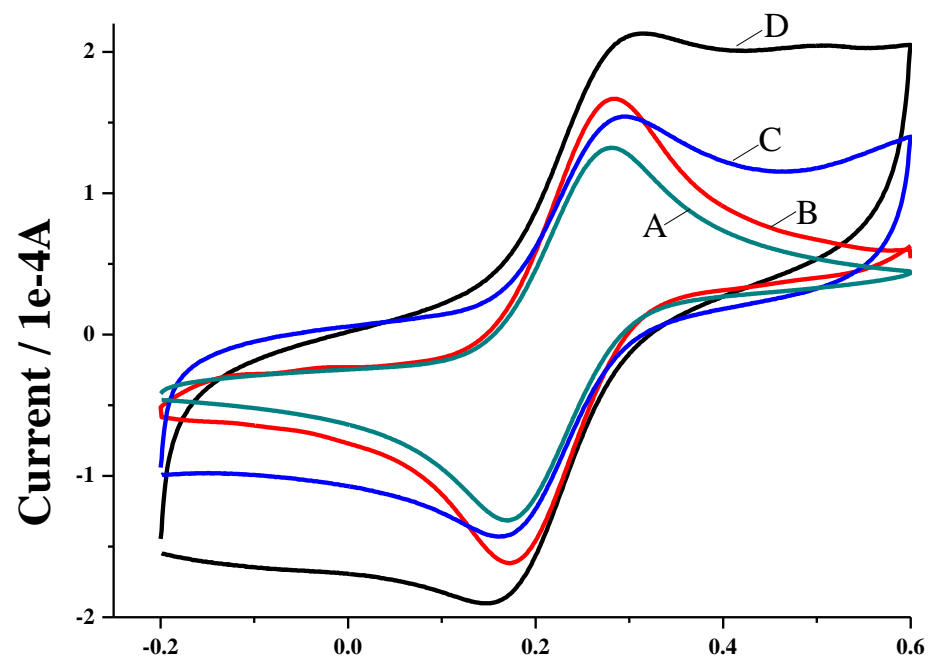

Potential / V

Meanwhile, the larger background current of the poly(4-ABA)/GCE (Figure 4C) than that of the bare GCE, implies the enhancement of the apparent area by the polymer. However, it is strange that a lower peak current at the poly(4-ABA)/GCE (about 11\%) than that of the bare GCE was found, which might be ascribed to the electrostatic repulsion between polymer and probe. Herein, since the $\mathrm{pK}_{\mathrm{a}}$ of 4ABA is about 3.0 [24], carboxylic groups of the polymer are negatively charged due to the dissociation 
in neutral surroundings. Therefore, the poly(4-ABA) would repel $\mathrm{Fe}(\mathrm{CN})_{6}{ }^{3-}$ arriving at the electrode surface, and led to the observed current decrease.

Figure 5 presents the EIS spectra obtained at the bare GCE (A), the MWNTs/GCE (B), poly(4-ABA)/GCE (C), and poly(4-ABA)/MWNTs/GCE (D). Plot B was a straight line, suggesting a fast electron transfer promoted by MWNTs. Plots A, C and D included a small semicircular part and a linear part, where the semicircular part at higher frequencies corresponded to the electron-transfer limited process, and the linear part at lower frequencies corresponded to the diffusion process.

Figure 5. EIS plots of bare GCE (A), MWNTs/GCE (B), poly(4-ABA)/GCE (C), and poly(4-ABA)/MWNTs/GCE (D). Frequency used: $0.05 \mathrm{~Hz}$ to $10 \mathrm{KHz}$; solution: $5 \mathrm{mM}$ $\mathrm{K}_{3} \mathrm{Fe}(\mathrm{CN})_{6} / \mathrm{K}_{4} \mathrm{Fe}(\mathrm{CN})_{6}$.

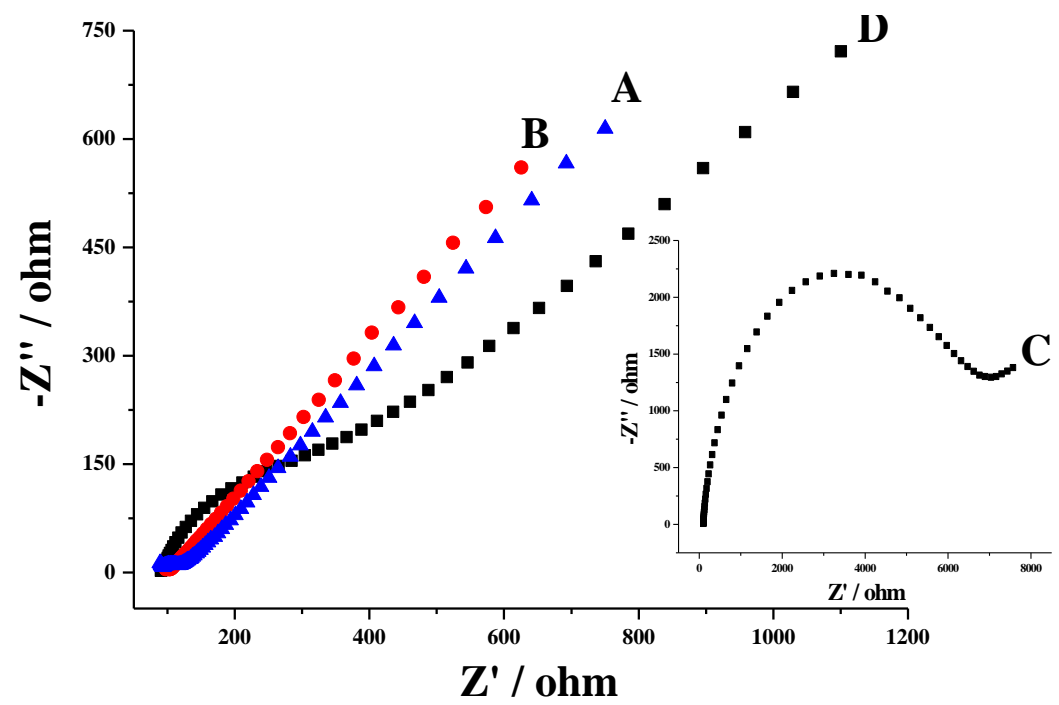

The resistance of electron transfer for GCE, poly(4-ABA)/GCE and poly(4-ABA)/MWNTs/GCE are $48.8 \Omega, 6,551 \Omega$, and $440.0 \Omega$, respectively. The clear increase of electron-transfer resistance at the poly(4-ABA)/GCE implies the modification of poly(4-ABA), which will block the electron-transfer of $\mathrm{Fe}(\mathrm{CN})_{6}{ }^{3-/ 4-}$ at the electrode due to the electrostatic repulsion between polymer and probe. This result is consistent with the previous cyclic voltammetry result. However, the electron-transfer resistance at poly(4-ABA)/MWNTs/GCE decreased obviously, which indicates the enhancement of conductivity by modification of the poly(4-ABA)/MWNTs composite film.

\subsection{Electrochemical responses of the poly(4-ABA)/MWNTs/GCE}

Figure 6 shows the electrochemical behavior of doxepin at the bare GCE (A), the poly(4-ABA)/GCE (B), the MWNTs/GCE (C), and the poly(4-ABA)/MWNTs/GCE (D). When the MWNTs or the poly(4-ABA) was introduced on the GCE, the current signal was amplified over 10-fold compared with that of the bare GCE, while the poly(4-ABA)/MWNTs/GCE exhibited the best anodic peak current among the four electrodes. The excellent sensitivity of the poly(4-ABA)/MWNTs/GCE could be ascribed to the presence of the nanocomposite film of MWNTs and poly(4-ABA), which might present excellent electrocatalytic activities and preconcentration towards doxepin. Furthermore, the oxidation potential of doxepin at the poly(4-ABA)/MWNTs/GCE 
was more negative compared with that of the MWNTs/GCE and the poly(4-ABA)/GCE, which also indicates the nice conductivity and the composite effect of the nanocomposite film. Additionally, no cathodic peak of doxepin was found on both bare GCE and modified electrodes, which suggests that the electrochemical oxidation of doxepin is irreversible.

Figure 6. Cyclic voltammograms of $1.0 \times 10^{-5} \mathrm{M}$ doxepin at bare GCE (A), poly(4-ABA)/GCE (B), MWNTs/GCE (C), and poly(4-ABA)/MWNTs/GCE (D). Scan rate: $100 \mathrm{mV} \mathrm{s}^{-1}$; supporting electrolyte: $0.10 \mathrm{M}$ PBS (pH 6.2); accumulation time: $60 \mathrm{~s}$.

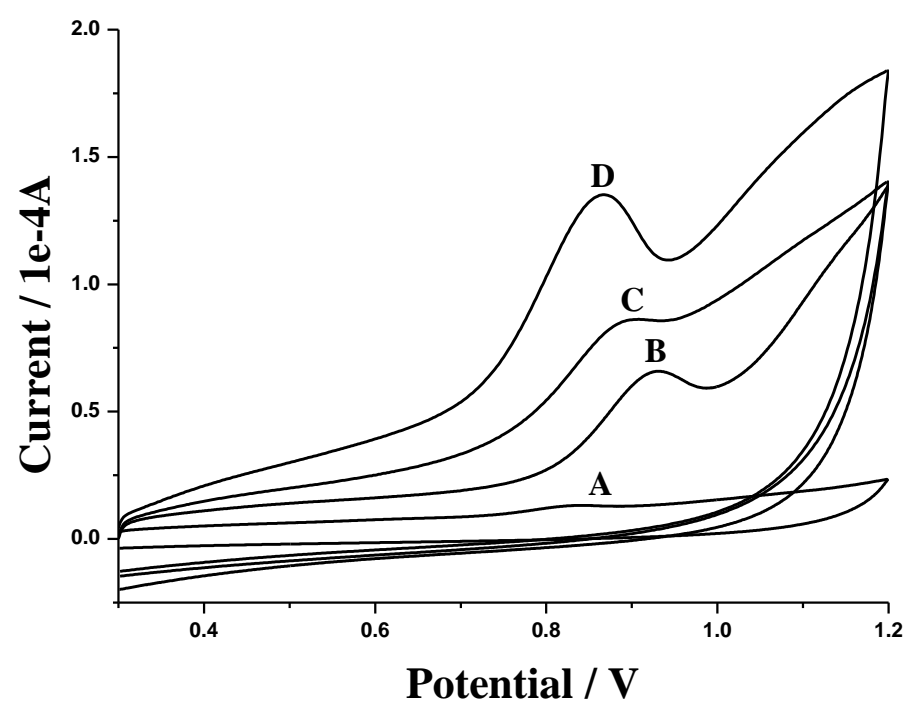

\subsection{Effect of scan rate}

With scan rate increasing, the anodic peak of doxepin grew. It was found that the anodic peak current was linear to the scan rate in the range of $10-200 \mathrm{mV} \mathrm{s}^{-1}$, the regression equation was:

$$
I_{p}=1.010+0.657 v\left(I_{p}(\mu A), v\left(\mathrm{mV} \mathrm{s}^{-1}\right), r=0.999\right)
$$

This indicates the electrode process is adsorption-controlled. In additional, the anodic peak potential and the logarithm of scan rate also showed a linear relationship, following the equation:

$$
E_{p}=0.712+0.0299 \operatorname{lnv}\left(E_{p}(V), r=0.998\right)
$$

According to the equation [25]:

$$
\mathrm{I}_{\mathrm{p}}=\mathrm{n}^{2} \mathrm{~F}^{2} \mathrm{vA} \Gamma_{0}^{*} / 4 \mathrm{RT}=\mathrm{nFQv} / 4 \mathrm{RT}
$$

the factor $\mathrm{n}$ was calculated to be 1.35 , which indicates one electron is lost during the electrochemical oxidation. This result is consistent with the mechanism of doxepin electrooxidation proposed in the literature [10]. The electrooxidation of doxepin might take place at the nitrogen atom in the alkylamine, resulting in the formation of a cation radical, followed by deprotonation. In addition, according to the equation [26]:

$$
\mathrm{E}_{\mathrm{p}}=\mathrm{E}^{\Phi}+[\mathrm{RT} /(1-\alpha) n \mathrm{~F}] \ln \left[\mathrm{RTk}_{\mathrm{s}} /(1-\alpha) \mathrm{nF}\right]+[\mathrm{RT} /(1-\alpha) \mathrm{nF}] \operatorname{lnv}
$$

the charge-transfer coefficient $\alpha$ was calculated to be 0.15 . 


\subsection{Optimization of the analytical conditions}

\subsubsection{Influence of amount of MWNTs}

As the volume of the MWNTs suspension was increased from 0 to $8 \mu \mathrm{L}$, the peak current response at the MWNTs/GCE was found to grow continuously. When the volume exceeded $8 \mu \mathrm{L}$, the response decreased. Apparently, the responses were closely related to the thickness of the MWNTs film. On the one hand, if the film was too thin, the number of adsorbed doxepin molecules was smaller, thus the response was smaller. On the other hand, when it was too thick, the film would become a little instable due to the desorption of MWNTs from the electrode surface, which led to the decrease of the peak current. Consequently, $8 \mu \mathrm{L}$ MWNTs suspension was chosen for modification.

\subsubsection{Influence of electropolymerization conditions}

The potential range for electropolymerization was very important for the preparation of the poly(4-ABA). The results showed that if the positive potential was lower than $+1.5 \mathrm{~V}$ or the negative potential was higher than $-0.8 \mathrm{~V}$, the polymerization reaction hardly occurred. When the positive potential reached $+1.8 \mathrm{~V}$, the electropolymerization happened. It was found that, the polymer prepared between the potential window from -1.4 to $+2.4 \mathrm{~V}$ exhibited much better electrochemical response to doxepin than the other ranges, thus the window was selected from -1.4 to $+2.4 \mathrm{~V}$.

According to the mechanism of the electropolymerization reaction of 4-ABA [23], a basic medium would be beneficial to the formation of the polymer. Thus, $0.10 \mathrm{M}$ buffer solutions (pH 7.5) of PBS, Tris- $\mathrm{HCl}, \mathrm{Na}_{2} \mathrm{~B}_{4} \mathrm{O}_{7}$ and $\mathrm{NH}_{3}-\mathrm{NH}_{4} \mathrm{Cl}$ were initially examined. The result showed that the responses did not differ, indicating the medium had little effect on the electropolymerization. Furthermore, when the $\mathrm{pH}$ value of $0.10 \mathrm{M}$ PBS varied from 7.0 to 9.0 , the response at $\mathrm{pH} 7.5$ was a little better than the other $\mathrm{pH}$. Generally, 0.10 M PBS ( $\mathrm{pH} 7.5$ ) was selected as the polymerization medium for its mildness.

In addition, when the oxygen dissolved in the medium was removed before electropolymerization, the doxepin responses remained almost unchanged. This suggests oxygen is not involved in the polymerization reaction, which is consistent with the electropolymerization mechanism of 4-ABA, so it was generally not necessary to remove oxygen before electropolymerization.

Moreover, when the number of the polymerization cycles was increased from 0 to 25 scans, it was found that the current responses of doxepin grew until 10 scans, then they reached a plateau, and trended to decrease slightly for more scans. This suggests the modified GCE has the best electrochemical behavior for 10 scans. More scans would produce a denser film and the film became insulate. Therefore, 10 scans were selected as the best number of polymerization cycles.

\subsubsection{Influence of $\mathrm{pH}$ and buffer solution}

As shown in Figure 7A, within the range from $\mathrm{pH} 4.2$ to 10.7 , the anodic peak current of doxepin increased until it attained the maximum at $\mathrm{pH}$ 6.2, then it decreased. Additionally, the anodic peak potential shifted negatively with the $\mathrm{pH}$ increasing (Figure $7 \mathrm{~B}$ ), following the linear equation:

$$
E_{p}=1.276-0.0671 p H\left(E_{p}(V), r=0.995\right)
$$


This implies that $\mathrm{H}^{+}$is involved in the oxidation of doxepin. According to literature [27], the $\mathrm{pK}_{\mathrm{a}}$ of doxepin is 9.0. At much lower pHs, the oxidation generally became difficult due to the strong protonation and responses were lower. As the $\mathrm{pH}$ grew, the responses were enhanced due to the deprotonation. Nevertheless, neutral doxepin is hydrophobic [28], and as the $\mathrm{pH}$ increased further and exceeded the $\mathrm{pK}_{\mathrm{a}}$, the solubility decreased gradually and drug precipitation occurred, which could affect the accumulation and caused the decrease of the responses. Therefore, $\mathrm{pH} 6.2$ was chosen as the optimum $\mathrm{pH}$ value. Then several supporting electrolytes including PBS, Tris- $\mathrm{HCl}, \mathrm{Na}_{2} \mathrm{~B}_{4} \mathrm{O}_{7}-\mathrm{HCl}$ and HAc-NaAc were also tested. The results showed that the response obtained in PBS was a little better than that in other media. Besides, when the buffer concentration varied from 0.02 to $0.4 \mathrm{M}$, no obvious effect on the responses was observed. Hence, 0.10 M PBS (pH 6.2) was chosen as the buffer solution.

Figure 7. Influence of $\mathrm{pH}$ on anodic peak current (A) and peak potential (B) of doxepin. Supporting electrolyte: 0.10 M PBS. Other conditions as in Figure 6.

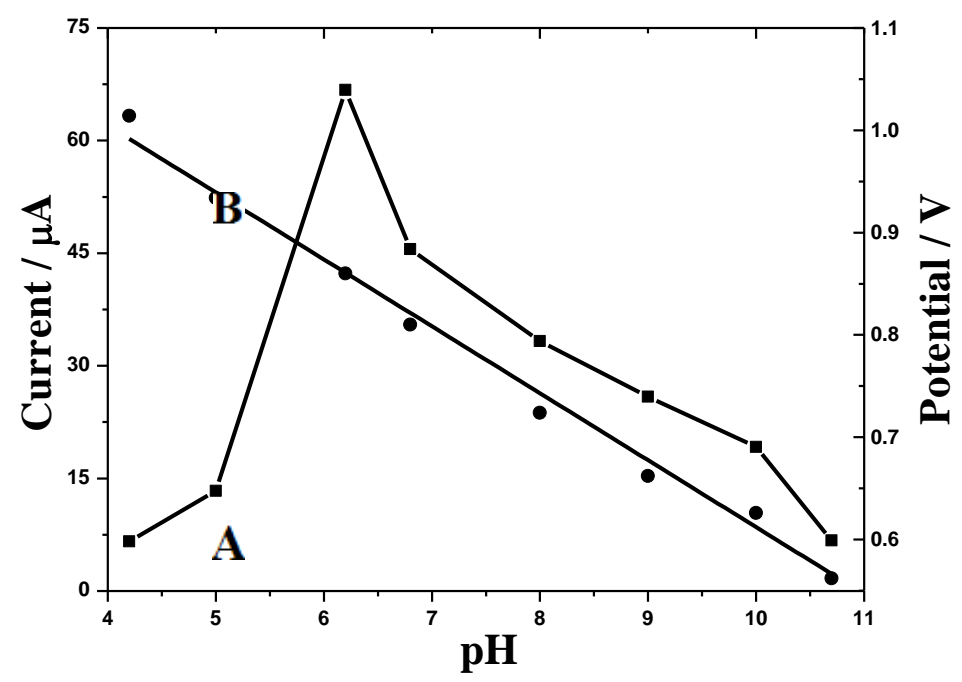

\subsubsection{Influence of accumulation time and potential}

For a $1 \times 10^{-5} \mathrm{M}$ doxepin solution, the anodic peak currents increased as the accumulation time increased. After $60 \mathrm{~s}$ of preconcentration, the responses reached the maximum, and longer accumulation time exhibited no more current growth, which implies the accumulation reaches saturation. Therefore, $60 \mathrm{~s}$ was chosen as accumulation time. When the accumulation potential varied from -0.6 to $+0.4 \mathrm{~V}$, the peak current decreased continuously. However, compared with at open circuit, the effect of accumulation potential was negligible. Hence, open circuit accumulation was adopted.

\subsection{Calibration curve}

Under the optimal analytical conditions, the determination of doxepin at different concentrations was performed. The anodic peak current was linear to the logarithm of the concentration of doxepin from $1.0 \times 10^{-9}$ to $1.0 \times 10^{-6} \mathrm{M}$. The linear regression equation was expressed as:

$$
\mathrm{i}_{\mathrm{p}}=2.379+0.189 \log \mathrm{C}\left(\mathrm{i}_{\mathrm{p}}(\mu \mathrm{A}), \mathrm{C}(\mathrm{M}), \mathrm{r}=0.997\right)
$$


The detection limit for doxepin was estimated to be $1.0 \times 10^{-10} \mathrm{M}(\mathrm{S} / \mathrm{N}=3)$, lower than that obtained by the boron-doped diamond electrode [16] and other chromatographic methods [7,29].

\subsection{The reproducibility, regeneration, stability and selectivity of poly(4-ABA)/MWNTs/GCE}

For eight parallel measurements of $1.0 \times 10^{-5} \mathrm{M}$ doxepin, the R.S.D. of the peak current was calculated to be $2.5 \%$. The electrode could be easily regenerated by repetitive cycling in a blank solution of $0.10 \mathrm{M}$ PBS $(\mathrm{pH}$ 6.2) for several times. The response of doxepin at the poly(4-ABA)/MWNTs/GCE could retain around $90 \%$ of the original response after seven days. This suggests the modified electrode has high stability.

The influence of foreign compounds was also tested at the poly(4-ABA)/MWNTs/GCE. It was found that several kinds of surfactants such as sodium dodecyl sulfate did not interfere, while Tween-20 and Triton X-100 could decrease the peak current. 50-fold $\mathrm{Cu}^{2+}, \mathrm{Ag}^{+}, \mathrm{Fe}^{3+}, 100$-fold epinephrine, vitamin $\mathrm{C}$, antipyrine, norfloxacin, allopurinol, thiamine, glucose, phenylalanine, captopril, or hydroxyzine, had no obvious effect on the determination. This suggests the poly(4-ABA)/MWNTs/GCE has certain resistance to some interferences.

\subsection{Applications}

The poly(4-ABA)/MWNTs/GCE was applied to detect doxepin in drug tablets. The pretreatment and determination procedures for doxepin tablets were the same as described in Section 2. The analytical results were shown in Table 1, and the recovery was 98.0-100.4\%. The doxepin content was calculated to be $24.7 \mathrm{mg}$ per tablet (its declared content was $25 \mathrm{mg}$ ). In addition, the accuracy of this method was examined by comparison with the result from the UV assay proposed by the Chinese Pharmacopoeia (the UV absorbance of doxepin at $297 \mathrm{~nm}$ in methanol solution containing $0.01 \mathrm{M} \mathrm{HCl}$ was chosen as the quantitative criterion) [28]. By means of F-test and t-test, the calculated F-value (2.71) and t-value (0.56) were both smaller than theoretical values (5.05 and 2.23, respectively, at the $95 \%$ confidence level). It implies that the method proposed could be reliably used for routine analysis.

Table 1. Measurement results of doxepin in drug tablets $(n=6)$.

\begin{tabular}{|c|cccccc|}
\hline $\begin{array}{c}\text { Sample } \\
\text { number }\end{array}$ & $\begin{array}{c}\text { Added } \\
(\boldsymbol{\mu} \mathbf{g} / \mathbf{m L})\end{array}$ & $\begin{array}{c}\text { Expected } \\
(\boldsymbol{\mu} \mathbf{g} / \mathbf{m L})\end{array}$ & $\begin{array}{c}\text { Found } \\
(\boldsymbol{\mu} \mathbf{g} / \mathbf{m L})\end{array}$ & $\begin{array}{c}\text { R.S.D. } \\
(\boldsymbol{\%})\end{array}$ & $\begin{array}{c}\text { Recovery } \\
(\boldsymbol{\%})\end{array}$ & $\begin{array}{c}\text { UV method } \\
(\boldsymbol{\mu g} / \mathbf{m L})\end{array}$ \\
\hline 0 & 0 & 1.00 & 0.99 & 2.5 & 99.0 & 0.98 \\
1 & 1.00 & 2.00 & 1.96 & 2.9 & 98.0 & 1.99 \\
2 & 2.00 & 3.00 & 2.97 & 2.1 & 99.0 & 2.98 \\
3 & 3.00 & 4.00 & 3.94 & 3.3 & 98.5 & 4.02 \\
4 & 4.00 & 5.00 & 5.02 & 1.7 & 100.4 & 4.99 \\
\hline
\end{tabular}

\section{Conclusions}

A sensitive electrochemical sensor for doxepin was prepared on GCE via modification of the nanocomposite film of the MWNTs and the poly(4-ABA). The excellent performance of the 
poly(4-ABA)/MWNTs/GCE could be ascribed to the effective preconcentration capacity and the excellent catalytic activities toward doxepin by the nanocomposite material. Thus, a sensitive detection method of doxepin due to the anodic peak was proposed. The as-prepared sensor with high sensitivity, speed, simplicity and low costs met the requirements of doxepin detection in drug tablets, and the procedure was proven to be reliable and could potentially be applied in clinical analysis of doxepin in physiological samples.

\section{Acknowledgements}

This work was supported by Shanghai Leading Academic Discipline Project (Project Number: B109 and 08XD14009), the NSFC (20675018 and 20892022), CSC, CSTC 2007BB5260 and Fudan University.

\section{References}

1. Chlobowska, Z.; Chudzikiewicz, E.; Koscienlniak, P.; Piekoszewski, W.; Stolarz, A. Application of Gas Chromatography with NPD Detection to the Analysis of Tricyclic Antidepressants in Blood. Chem. Anal. 2003, 48, 255-263.

2. Uddin, M.N.; Samanidou, V.F.; Papadoyannis, I.N. Development and Validation of an HPLC Method for the Determination of Benzodiazepines and Tricyclic Antidepressants in Biological Fluids after Sequential Spe. J. Sep. Sci. 2008, 31, 2358-2370.

3. Ruiz-Angel, M.J.; Carda-Broch, S.; Simo-Alfonso, E.F.; Garcia-Alvarez-Coque, M.C. Optimised Procedures for the Reversed-Phase Liquid Chromatographic Analysis of Formulations Containing Tricyclic Antidepressants. J. Pharm. Biomed. Anal. 2003, 32, 71-84.

4. Maslanka, A.; Krzek, J. Densitometric High Performance Thin-Layer Chromatography Identification and Quantitative Analysis of Psychotropic Drugs. J. AOAC Int. 2005, 88, 70-79.

5. Gronewold, A.; Dettling, A.; Haffner, H.T.; Skopp, G. Doxepin and Nordoxepin Concentrations in Body Fluids and Tissues in Doxepin Associated Deaths. Forensic. Sci. Int. 2009, 190, 74-79.

6. Hostetter, A.L.; Stowe, Z.N.; Cox, M.; Ritchie, J.C. A Novel System for the Determination of Antidepressant Concentrations in Human Breast Milk. Ther. Drug Monit. 2004, 26, 47-52.

7. Ma, Q.; Chen, M.; Shi, Z.G.; Feng, Y.Q. Preparation of a Poly(N-Isopropylacrylamide-Co-Ethylene Dimethacrylate) Monolithic Capillary and Its Application for in-Tube Solid-Phase Microextraction Coupled to High-Performance Liquid Chromatography. J. Sep. Sci. 2009, 32, 2592-2600.

8. Hummel, D.; Loffler, D.; Fink, G.; Ternes, T.A. Simultaneous Determination of Psychoactive Drugs and Their Metabolites in Aqueous Matrices by Liquid Chromatography Mass Spectrometry. Environ. Sci. Technol. 2006, 40, 7321-7328.

9. Kou, H.S.; Chen, C.C.; Huang, Y.H.; Ko, W.K.; Wu, H.L.; Wu, S.M. Method for Simultaneous Determination of Eight Cyclic Antidepressants by Cyclodextrin-Modified Capillary Zone Electrophoresis: Applications in Pharmaceuticals. Anal. Chim. Acta 2004, 525, 23-30.

10. Greenway, G.M.; Dolman, S.J.L. Analysis of Tricyclic Antidepressants Using Electrogenerated Chemiluminescence. Analyst 1999, 124, 759-762. 
11. Wu, C.K.; Feng, S.L.; Jing, F. Spectrometric Study on the Interaction of Doxepin Hydrochloride and Fast Green and Its Application. Spectrosc. Spectr. Anal. 2007, 27, 2490-2493.

12. Georgiou, M.E.; Georgiou, C.A.; Koupparis, M.A. Rapid Automated Spectrophotometric Competitive Complexation Studies of Drugs with Cyclodextrins Using the Flow Injection Gradient Technique: Tricyclic Antidepressant Drugs with Alpha-Cyclodextrin. Analyst 1999, 124, 391-396.

13. Acedo-Valenzuela, M.I.; Galeano-Diaz, T.; Mora-Diez, N.; Silva-Rodriguez, A. Response Surface Methodology for the Optimisation of Flow-Injection Analysis with in Situ Solvent Extraction and Fluorimetric Assay of Tricyclic Antidepressants. Talanta 2005, 66, 952-960.

14. Chodkowski, J.; Chrzanowski, A.; Gralewskaludwicka, D. Polarographic-Determination of Doxepin Hydrochloride. Chem. Anal. 1992, 37, 629-633.

15. Hopkala, H.; Zareba, S. Doxepin Ion-Selective Membrane Electrode, Preparation and Use in Pharmaceutical Analysis. Chem. Anal. 1996, 41, 413-417.

16. Ivandini, T.A.; Sarada, B.V.; Terashima, C.; Rao, T.N.; Tryk, D.A.; Ishiguro, H.; Kubota, Y.; Fujishima, A. Electrochemical Detection of Tricyclic Antidepressant Drugs by HPLC Using Highly Boron-Doped Diamond Electrodes. J. Electroanal. Chem. 2002, 521, 117-126.

17. Huang, F.; Peng, Y.Y.; Jin, G.Y.; Zhang, S.; Kong, J.L. Sensitive Detection of Haloperidol and Hydroxyzine at Multi-Walled Carbon Nanotubes-Modified Glassy Carbon Electrodes. Sensors 2008, 8, 1879-1889.

18. Jin, G.Y.; Zhang, Y.H.; Cheng, W.X. Poly(P-Aminobenzene Sulfonic Acid)-Modified Glassy Carbon Electrode for Simultaneous Detection of Dopamine and Ascorbic Acid. Sens. Actuator. B-Chem. 2005, 107, 528-534.

19. Huang, F.; Jin, G.Y.; Liu, Y.; Kong, J.L. Sensitive Determination of Phenylephrine and Chlorprothixene at Poly(4-Aminobenzene Sulfonic Acid) Modified Glassy Carbon Electrode. Talanta 2008, 74, 1435-1441.

20. Jin, G.Y.; Huang, F.; Kong, J.L. Sensitive Determination of Clomipramine at Poly-ABSA/Pt Nano-Clusters Modified Glassy Carbon Electrode. Anal. Lett. 2007, 40, 3392-3404.

21. Jin, G.Y.; Huang, F.; Li, W.; Yu, S.N.; Zhang, S.; Kong, J.L. Sensitive Detection of Trifluoperazine Using a Poly-ABSA/SWNTs Film-Modified Glassy Carbon Electrode. Talanta 2008, 74, 815-820.

22. Xu, F.; Gao, M.N.; Wang, L.; Shi, G.Y.; Zhang, W.; Jin, L.T.; Jin, J.Y. Sensitive Determination of Dopamine on Poly(Aminobenzoic Acid) Modified Electrode and the Application toward an Experimental Parkinsonian Animal Model. Talanta 2001, 55, 329-336.

23. Xu, F.; Gao, M.N.; Shi, G.Y.; Wang, L.; Zhang, W.; Xue, J.; Jin, L.T.; Jin, J.Y. Simultaneous Detection of Monoamines in Rat Striatal Microdialysate at Poly(Para-Aminobenzoic Acid) Modified Electrode by High-Performance Liquid Chromatography. Anal. Chim. Acta 2001, 439, 239-246.

24. Liu, J.Y.; Cheng, L.; Li, B.F.; Dong, S.J. Covalent Modification of a Glassy Carbon Surface by 4-Aminobenzoic Acid and Its Application in Fabrication of a Polyoxometalates-Consisting Monolayer and Multilayer Films. Langmuir 2000, 16, 7471-7476. 
25. Bard, A.J.; Faulkner, L.R. Electrochemical Methods: Fundamentals and Applications; John Wiley \& Sons: New York, NY, USA, 1980; p. 595.

26. Laviron, E. General Expression of the Linear Potential Sweep Voltammogram in the Case of Diffusionless Electrochemical Systems. J. Electroanal. Chem. 1979, 101, 19-28.

27. Dell'Aquila, C. Separation of Tricyclic Antidepressants by Capillary Zone Electrophoresis with N,N,N',N'-Tetramethyl-1,3-Butanediamine (TMBD) as an Effective Electrolyte Additive. J. Pharm. Biomed. Anal. 2002, 30, 341-350.

28. Chinese Pharmacopoeia Committee. Chinese Pharmacopoeia (Part II), 1st ed.; Chemical Industry Press: Beijing, China, 2005; pp. 505-506.

29. Li, J.G.; Zhao, F.J.; Ju, H.X. Simultaneous Determination of Psychotropic Drugs in Human Urine by Capillary Electrophoresis with Electrochemiluminescence Detection. Anal. Chim. Acta. 2006, 575, 57-61.

(C) 2010 by the authors; licensee MDPI, Basel, Switzerland. This article is an open access article distributed under the terms and conditions of the Creative Commons Attribution license (http://creativecommons.org/licenses/by/3.0/). 